\title{
Selective Convergent Synthesis of Aliphatic Polyurethane Dendrimers
}

W. James Feast ${ }^{\dagger}$, Steve P. Rannard* and Alison Stoddart

\section{Supplementary Information}

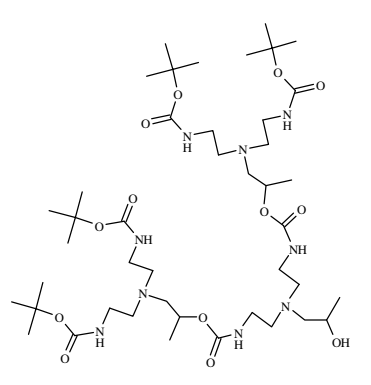

G2-[t-Bu-AEAP 2$] \mathrm{OH}$

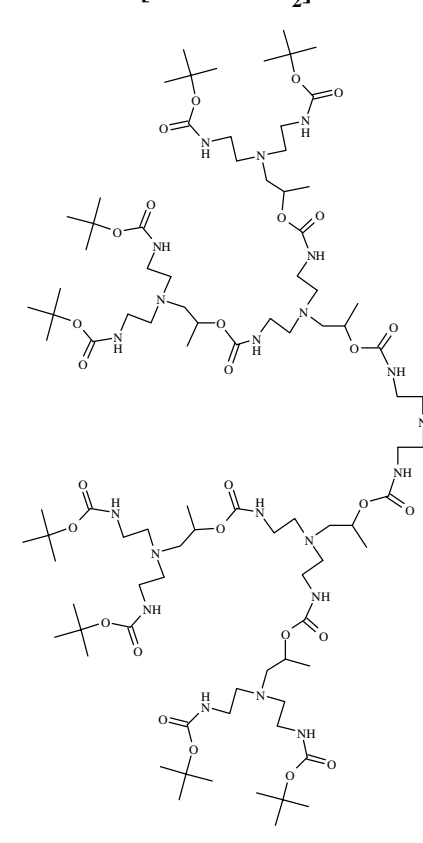

G3-[t-Bu-AEAP 3 ]OH

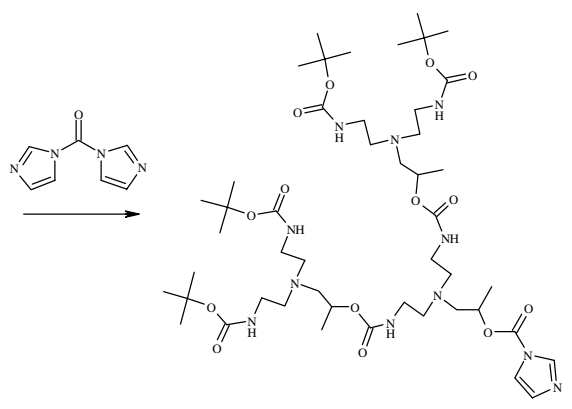

G2-[t-Bu-AEAP 2$]$ Im

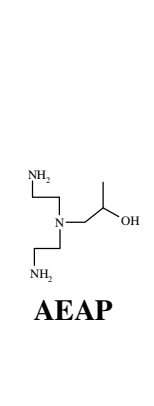

Synthesis of G3-[t-Bu-AEAP $\mathbf{3}] \mathbf{O H}$ :

CDI (4.62 mmol) was added to a stirred solution of G2-[t-Bu-AEAP $\mathbf{2}] \mathbf{O H , ~ ( 3 . 8 9 ~}$ $\mathrm{mmol})$ in toluene $(50 \mathrm{~mL})$. The mixture was heated at $60^{\circ} \mathrm{C}$ for $4 \mathrm{hrs}$. Subsequently, the reaction mixture was analysed by ${ }^{1} \mathrm{H}$ NMR spectroscopy and interpretation of the spectrum indicated there was no evidence of the starting materials (i.e. G2- $[t-\mathrm{Bu}-$ 
$\left.\mathrm{AEAP}_{2}\right] \mathrm{OH}$ or $\left.\mathrm{CDI}\right)$. The branching unit AEAP $(1.95 \mathrm{mmol})$ was added and the solution was heated for 1 day at $60^{\circ} \mathrm{C}$. The reaction mixture was concentrated in vacuo and redissolved in $\mathrm{CH}_{2} \mathrm{Cl}_{2}(100 \mathrm{~mL})$. The organic phase was subsequently washed with water ( 3 x $100 \mathrm{~mL}$ ), dried over $\mathrm{MgSO}_{4}$ and the solvent removed using the rotary evaporator. The oil obtained was purified by column chromatography (silica gel, eluting with EtOAc increasing to EtOAc:MeOH, 100:5) and then purified by preparative GPC (Biobeads, eluting with toluene) to give compound G3-[t-Bu$\left.\mathbf{A E A P}_{3}\right] \mathbf{O H}$ as a colourless amorphous solid $(48 \%) . \mathrm{T}_{\mathrm{g}}=40.0^{\circ} \mathrm{C}$. Found $\mathrm{C}, 54.16$;

$\mathrm{H}, 9.08 ; \mathrm{N}, 13.83 \% . \quad \mathrm{C}_{95} \mathrm{H}_{185} \mathrm{O}_{29} \mathrm{~N}_{21}$ requires, C, 54.71; H, 8.94; N, $14.10 \% .{ }^{13} \mathrm{C}$ NMR (62.9 MHz, $\left.\mathrm{CD}_{3} \mathrm{OD}\right) \delta$ 19.0, 21.0, 28.9, 39.6, 40.0, 55.6, 55.9, 61.0, 64.0, 66.0, 70.7, 79.9, 158.3, 158.7, 158.8. ${ }^{1} \mathrm{H}$ NMR (400 MHz, $\left.\mathrm{CD}_{3} \mathrm{OD}\right) \delta 1.12(\mathrm{~d}, \mathrm{~J}=6 \mathrm{~Hz}, 3 \mathrm{H})$, $1.20(\mathrm{~d}, \mathrm{~J}=6.4 \mathrm{~Hz}, 18 \mathrm{H}), 1.44(\mathrm{~s}, 72 \mathrm{H}), 2.41-2.64(\mathrm{~m}, 42 \mathrm{H}), 3.04-3.24(\mathrm{~m}, 28 \mathrm{H}), 3.78$ (m, 1H), 4.85 (m, obscured by water peak, 6H), 6.42 (s, br, $\mathrm{O}(\mathrm{CO}) \mathrm{NHCH}_{2} \mathrm{CH}_{2}$ ), 6.71 (s, br, $\mathrm{O}(\mathrm{CO}) \mathrm{NHCH}_{2} \mathrm{CH}_{2}$ ), 6.85 (s, br, $\mathrm{O}(\mathrm{CO}) \mathrm{NHCH}_{2} \mathrm{CH}_{2}$ ). $\quad \mathrm{m} / z$ (ES MS) 2108.2 $[\mathrm{M}+\mathrm{Na}]^{+}, 1054.4[\mathrm{M}+2 \mathrm{H}]^{2+}, 1065.4[\mathrm{M}+\mathrm{H}+\mathrm{Na}]^{2+} . \mathrm{m} / z$ (MALDI TOF (Voyager) MS) $2086[\mathrm{M}+\mathrm{H}]^{+}, 2108[\mathrm{M}+\mathrm{Na}]^{+}, 2124[\mathrm{M}+\mathrm{K}]^{+}$calculated $\mathrm{M}_{\mathrm{w}}=2085.61$. GPC; $\mathrm{M}_{\mathrm{w}}=$ $2300, \mathrm{Pd}=1.00$.

\section{${ }^{13}$ CNMR spectrum of G3-[t-Bu-AEAP 3 ] OH}

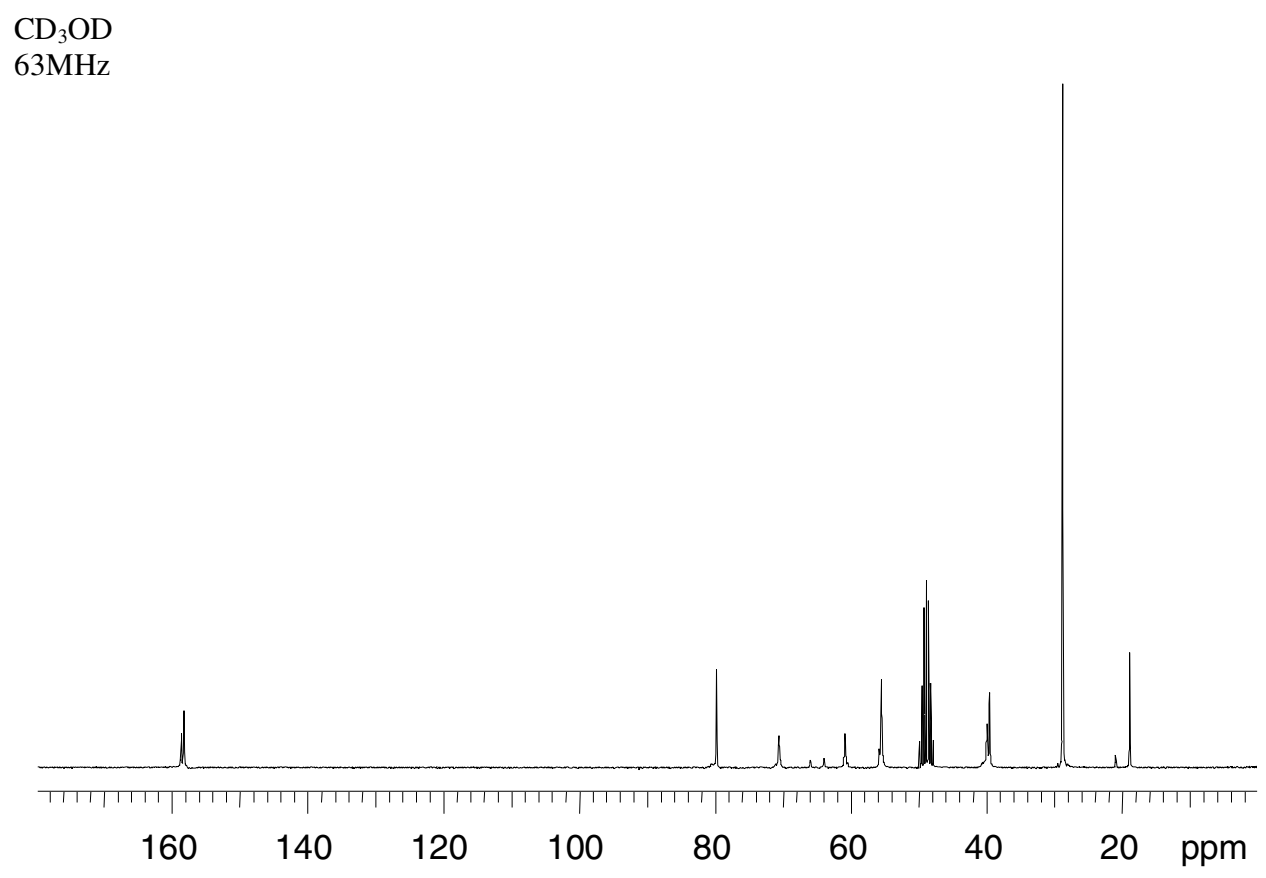




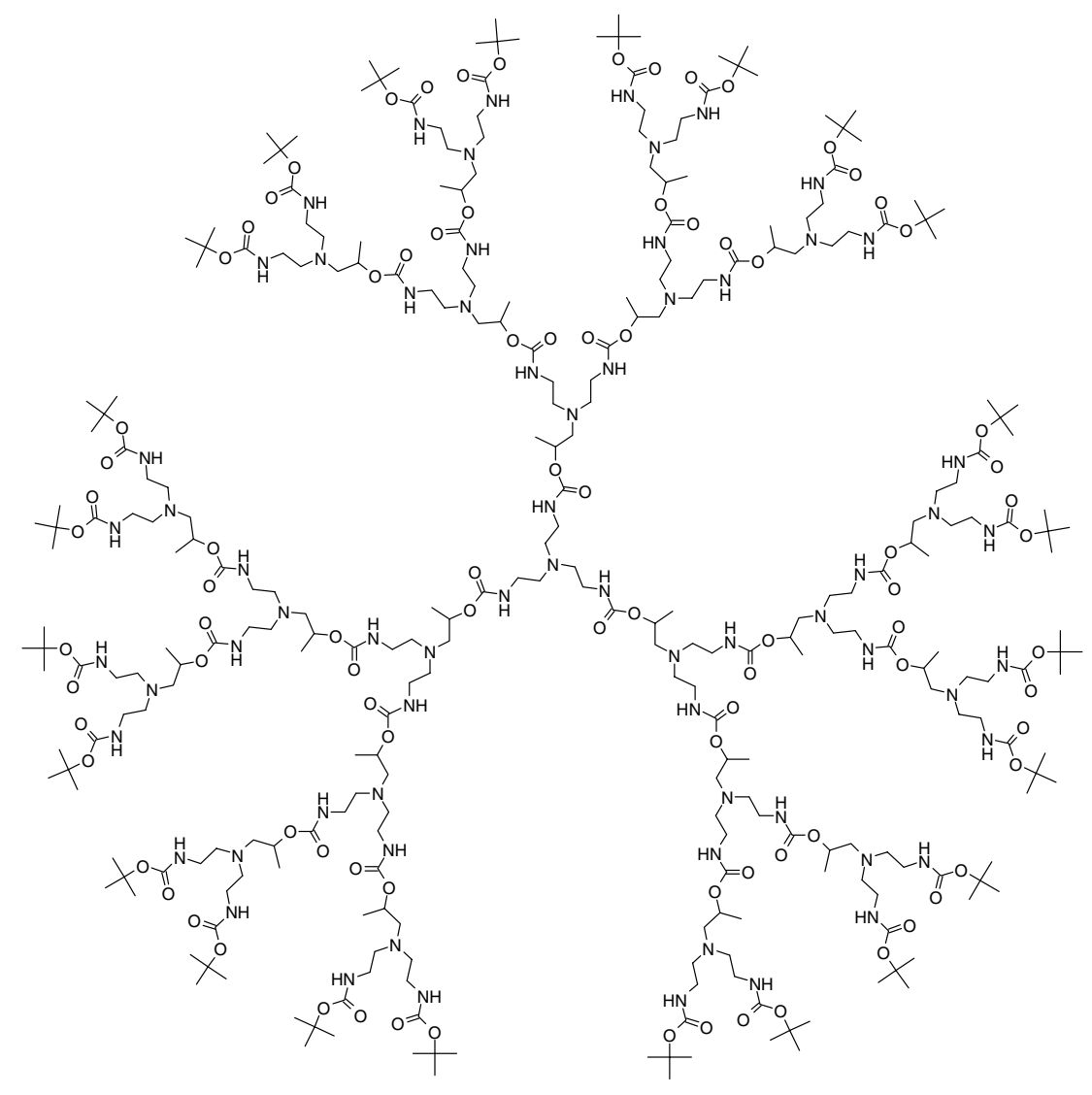

Synthesis of G3-[t-Bu-AEAP 3 TAEA:

CDI $(0.57 \mathrm{mmol})$ was added to a stirred solution of G3-[t-Bu-AEAP 3$] \mathrm{OH}(0.47$ $\mathrm{mmol})$ in toluene $(40 \mathrm{~mL})$ and the mixture was heated at $60^{\circ} \mathrm{C}$ for 4 hours. Subsequently, the reaction mixture was analysed by ${ }^{1} \mathrm{H}$ NMR spectroscopy and interpretation of the spectrum indicated no evidence of the presence of starting materials (i.e. G3-[t-Bu-AEAP 3$] \mathrm{OH}$ or $\mathrm{CDI})$. TAEA $(0.16 \mathrm{mmol})$ was added to the solution and the mixture was heated for 1 day at $60^{\circ} \mathrm{C}$. The reaction mixture was concentrated in vacuo and redissolved in $\mathrm{CH}_{2} \mathrm{Cl}_{2}(100 \mathrm{~mL})$. The organic phase was subsequently washed with water $(3 \times 100 \mathrm{~mL})$, dried over $\mathrm{MgSO}_{4}$ and the solvent removed using the rotary evaporator. The yellow oil obtained was purified by column chromatography (silica gel, eluting with EtOAc:MeOH 100:5 increasing to EtOAc:MeOH 100:10). The colourless oil obtained was purified further by preparative GPC (Biobeads, eluting with toluene) to give compound G3-[t-Bu- 
$\mathrm{AEAP}_{3}$ ]TAEA was isolated as a white amorphous solid $(20 \%) . \mathrm{T}_{\mathrm{g}}=48.9^{\circ} \mathrm{C}$. Found $\mathrm{C}, 54.46 ; \mathrm{H}, 8.74 ; \mathrm{N}, 13.41 \%$. $\mathrm{C}_{294} \mathrm{H}_{567} \mathrm{O}_{90} \mathrm{~N}_{67}$ requires, $\mathrm{C}, 54.48 ; \mathrm{H}, 8.82 ; \mathrm{N}$, 14.48\%. ${ }^{13} \mathrm{C}$ NMR (125 MHz, $\left.\mathrm{CD}_{3} \mathrm{OD}\right) \delta 19.1,29.0,39.9,40.2,55.6,55.7,61.1$, 70.9, 80.0, 158.3, 158.6. ${ }^{1} \mathrm{H}$ NMR $\left(400 \mathrm{MHz}, \mathrm{CD}_{3} \mathrm{OD}\right) \delta 1.20(\mathrm{~d}, \mathrm{~J}=6.4 \mathrm{~Hz}, 63 \mathrm{H})$, $1.44(\mathrm{~s}, 216 \mathrm{H}), 2.48-2.63(\mathrm{~m}, 132 \mathrm{H}), 3.05-3.25(\mathrm{~m}, 90 \mathrm{H}), 4.85$ (m, obscured by water peak, 21H), 6.42 (s, br, OC(O)NHCH$\left.{ }_{2}\right), 6.71$ (s, br, OC(O)NHCH$\left.{ }_{2}\right) . m / z(E S ~ M S)$ $3235.8[\mathrm{M}+2 \mathrm{H}]^{2+}, 3246.9[\mathrm{M}+\mathrm{H}+\mathrm{Na}]^{2+}, 3257.9[\mathrm{M}+2 \mathrm{Na}]^{2+} . \mathrm{m} / z(\mathrm{MALDI}$ TOF (Kratos) MS) $6469[\mathrm{M}+\mathrm{H}]^{+}$, calculated $\mathrm{M}_{\mathrm{w}}=6481.05$. GPC; $\mathrm{M}_{\mathrm{w}}=4980, \mathrm{Pd}=1.06$.

${ }^{13} \mathrm{C}$ NMR spectrum of third generation dendrimer $\mathrm{G} 3-[t$-Bu-AEAP 3$]$ TAEA

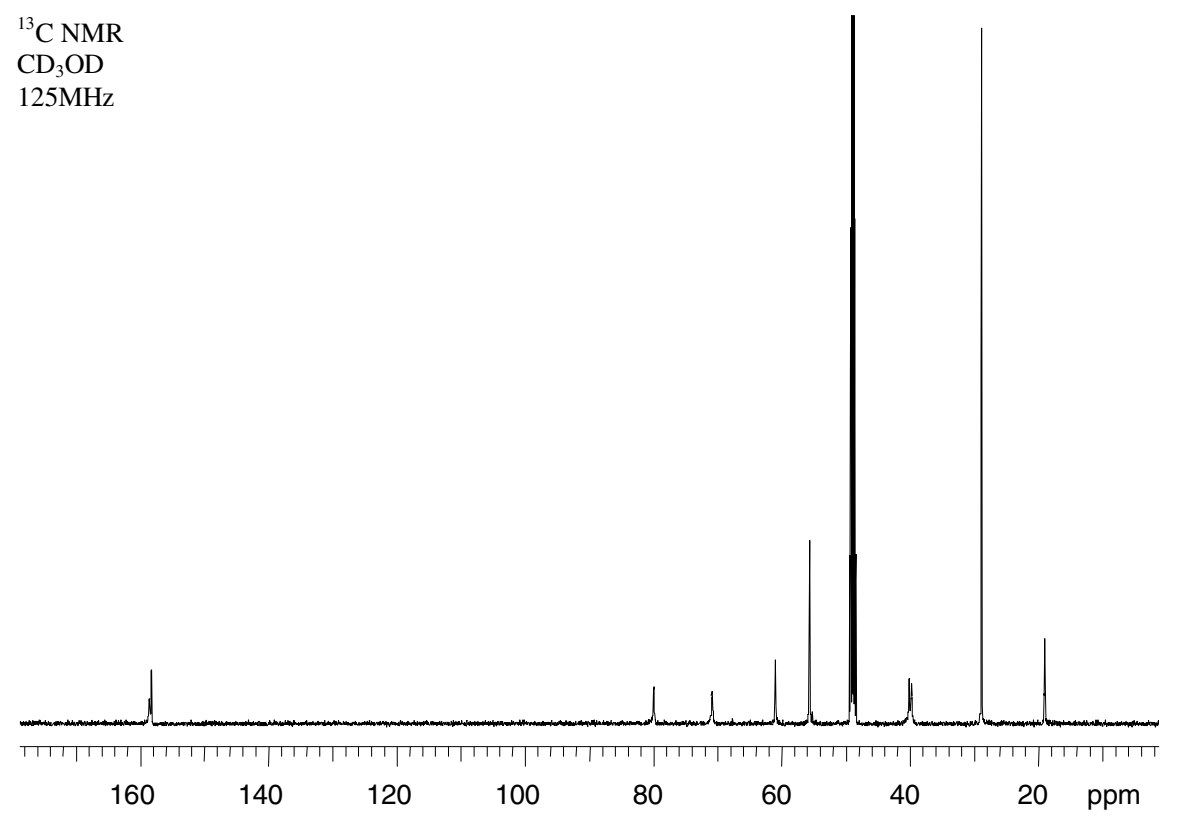




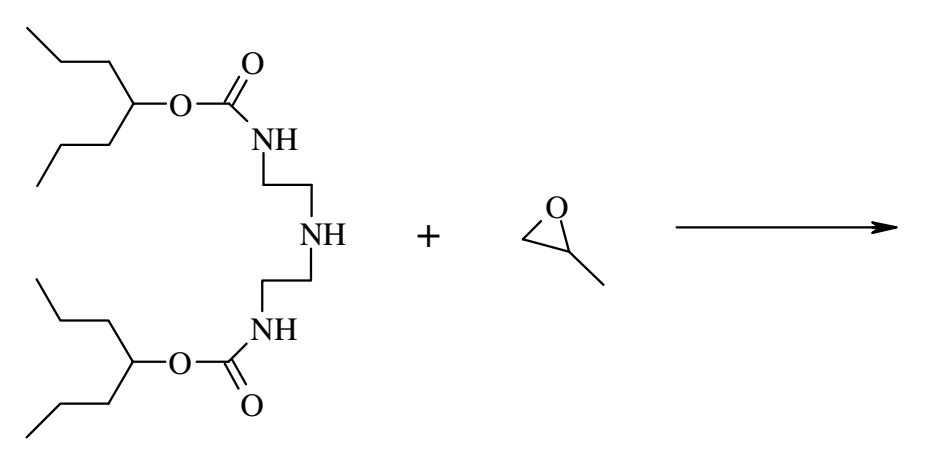

G1-[4-Hept-DETA]NH

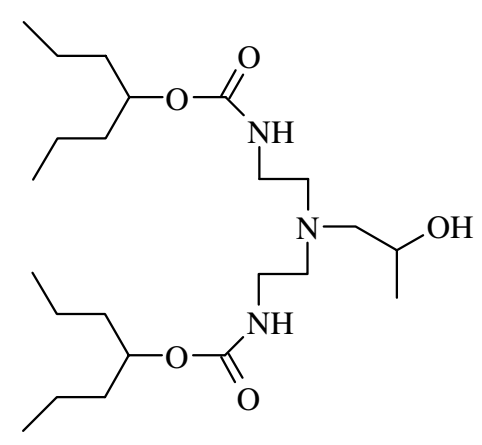

G1-[4-Hept-AEAP]OH

\section{Synthesis of G1-[4-Hept-AEAP]OH :}

To a stirred solution of G1-[4-Hept-DETA]NH (67.5 mmol) in ethanol, (200 mL) propylene oxide $\left(11.8 \mathrm{~g}, 202 \mathrm{mmol}\right.$ ) was added and the mixture heated at $30^{\circ} \mathrm{C}$ for 20 hrs. The solvent was removed using the rotary evaporator and the oil dried under vacuum $\left(10^{-1} \mathrm{mbar}\right)$ for 1 day. Purification by column chromatography (silica gel, eluting with EtOAc) gave G1-[4-Hept-AEAP]OH as a colourless oil (23.8 g, 91\%). $\mathrm{T}_{\mathrm{g}}=-39.5^{\circ} \mathrm{C}$. Found $\mathrm{C}, 61.90 ; \mathrm{H}, 10.61 ; \mathrm{N}, 9.43 \% . \mathrm{C}_{23} \mathrm{H}_{47} \mathrm{~N}_{3} \mathrm{O}_{5}$ requires, $\mathrm{C}, 61.99$; $\mathrm{H}, 10.63 ; \mathrm{N}, 9.43 \% .{ }^{13} \mathrm{C} \mathrm{NMR}\left(62.9 \mathrm{MHz}, \mathrm{CDCl}_{3}\right) \delta 14.0,18.5,20.0,36.7,39.2$, 55.1, 63.2, 64.1, 74.4, 157.2. ${ }^{1} \mathrm{H}$ NMR $\left(300 \mathrm{MHz}, \mathrm{CDCl}_{3}\right) \delta 0.91(\mathrm{t}, \mathrm{J}=7.2 \mathrm{~Hz}, 12 \mathrm{H})$, $1.12(\mathrm{~d}, \mathrm{~J}=6.3 \mathrm{~Hz}, 3 \mathrm{H}), 1.33(\mathrm{~m}, 8 \mathrm{H}), 1.47(\mathrm{~m}, 8 \mathrm{H}), 1.63(\mathrm{~s}, \mathrm{br}, \mathrm{OH}), 2.34$ (dd, $\mathrm{J}=12.9 \mathrm{~Hz}, \mathrm{~J}=9.9 \mathrm{~Hz}, 1 \mathrm{H}), 2.46(\mathrm{dd}, \mathrm{J}=13.2 \mathrm{~Hz}, \mathrm{~J}=3 \mathrm{~Hz}, 1 \mathrm{H}), 2.56(\mathrm{dt}, \mathrm{J}=13.2 \mathrm{~Hz}$, $\mathrm{J}=5.4 \mathrm{~Hz}, 2 \mathrm{H}), 2.67(\mathrm{~m}, 2 \mathrm{H}), 3.23(\mathrm{~m}, 4 \mathrm{H}), 3.73(\mathrm{~m}, 1 \mathrm{H}), 4.76(\mathrm{qn}, \mathrm{J}=6 \mathrm{~Hz}, 2 \mathrm{H}), 5.07$ (s, br, $\left.\mathrm{O}(\mathrm{CO}) \mathrm{NHCH}_{2}\right) . \mathrm{m} / \mathrm{z}(\mathrm{GC}, \mathrm{EI}) 446[\mathrm{M}+\mathrm{H}]^{+}$, calculated $\mathrm{M}_{\mathrm{w}}=445.64$.
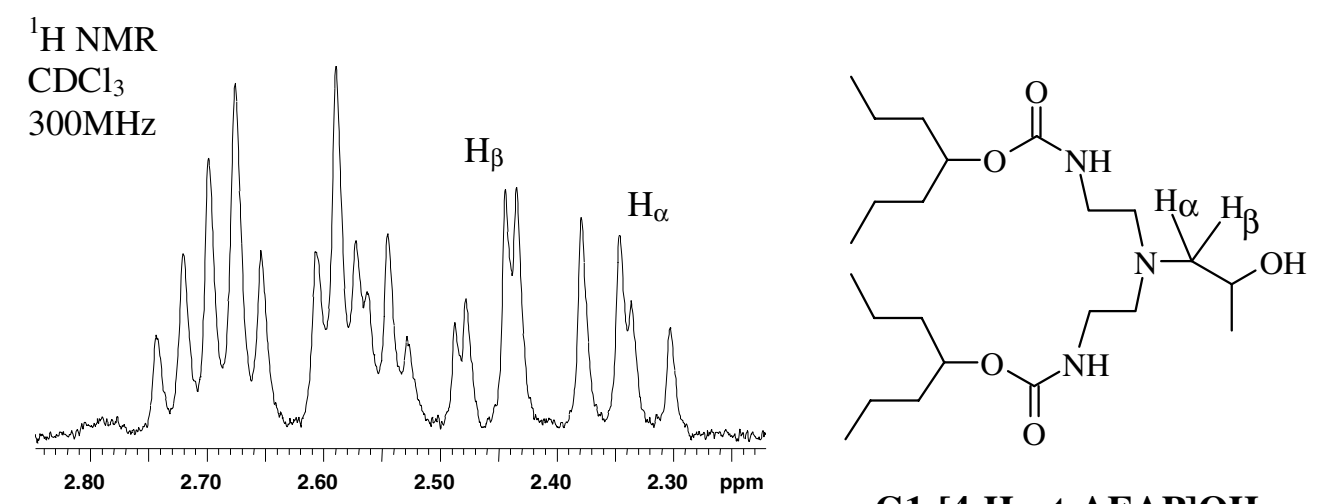

\section{G1-[4-Hept-AEAP]OH}

${ }^{1} \mathrm{H}$ NMR spectrum of G1-[4-Hept-AEAP]OH demonstrating coupling of diastereotopic hydrogens 


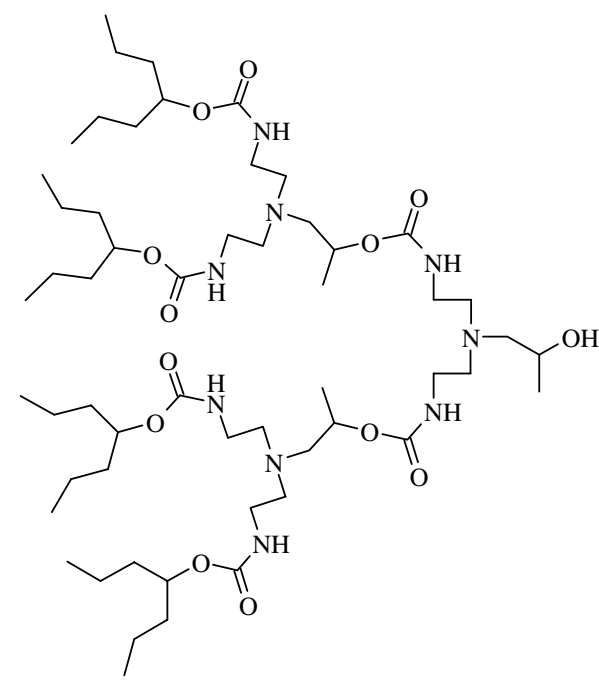

G2-[4-Hept-AEAP 2 ]OH

Synthesis of G2-[4-Hept-AEAP 2 ]OH:

CDI (28.1 mmol) was added to a stirred solution of G1-[4-Hept-AEAP]OH (24.4 $\mathrm{mmol})$ in toluene $(100 \mathrm{~mL})$. The mixture was heated at $60^{\circ} \mathrm{C}$ for $6 \mathrm{hrs}$. Subsequently, the reaction mixture was analysed by ${ }^{1} \mathrm{H}$ NMR spectroscopy and interpretation of the spectrum indicated there was no evidence of the starting materials (G1-[4-HeptAEAP]OH or CDI). The branching unit AEAP (13.4 mmol) was added and the solution was heated for 20 hrs. The reaction mixture was concentrated in vacuo and redissolved in $\mathrm{CH}_{2} \mathrm{Cl}_{2}(200 \mathrm{~mL})$. The organic phase was subsequently washed with water $(3 \times 250 \mathrm{~mL})$, dried over $\mathrm{MgSO}_{4}$ and the solvent removed using the rotary evaporator. The resulting pale yellow oil was purified by column chromatography (silica gel, eluting with EtOAc: $\mathrm{C}_{6} \mathrm{H}_{12}$ 1:23) and the colourless oil obtained was dried under vacuum $\left(10^{-1} \mathrm{mbar}\right)$ to give compound $\mathrm{G} 2$-[4-Hept-AEAP 2 ]OH as a sticky colourless oil $(49 \%) . \quad \mathrm{T}_{\mathrm{g}}=-4.3^{\circ} \mathrm{C}$. Found $\mathrm{C}, 59.54 ; \mathrm{H}, 9.91 ; \mathrm{N}, 11.20 \%$. $\mathrm{C}_{55} \mathrm{H}_{109} \mathrm{~N}_{9} \mathrm{O}_{13}$ requires, $\mathrm{C}, 59.81 ; \mathrm{H}, 9.95 ; \mathrm{N}, 11.41 \%$. ${ }^{13} \mathrm{C} \mathrm{NMR}\left(62.9 \mathrm{MHz}, \mathrm{CD}_{3} \mathrm{OD}\right)$ $\delta 14.5,18.9,19.5,20.9,37.8,39.9$ (resonances from two distinct carbons overlap), 55.5, 55.9, 60.9, 64.0, 65.8 (split into 3 peaks), 70.6, 74.9, 158.6, 158.9. ${ }^{1} \mathrm{H}$ NMR $\left(400 \mathrm{MHz}, \mathrm{CD}_{3} \mathrm{OD}\right) \delta 0.92(\mathrm{t}, \mathrm{J}=7.2 \mathrm{~Hz}, 24 \mathrm{H}), 1.12(\mathrm{~d}, \mathrm{~J}=6.4 \mathrm{~Hz}, 3 \mathrm{H}), 1.19$ (d, J=6.4 $\mathrm{Hz}, 6 \mathrm{H}), 1.30-1.41(\mathrm{~m}, 16 \mathrm{H}), 1.47-1.54(\mathrm{~m}, 16 \mathrm{H}), 2.38-2.68(\mathrm{~m}, 18 \mathrm{H}), 3.09-3.28(\mathrm{~m}$, 12H), $3.77(\mathrm{~m}, 1 \mathrm{H}), 4.73(\mathrm{~m}, 4 \mathrm{H}), 4.85(\mathrm{~m}, 2 \mathrm{H}), 6.67$ (s, br, $\left.\mathrm{O}(\mathrm{CO}) \mathrm{NHCH}_{2} \mathrm{CH}_{2}\right), 6.89$ (s, br, $\left.\mathrm{O}(\mathrm{CO}) \mathrm{NHCH}_{2} \mathrm{CH}_{2}\right) . \quad m / z$ (ES MS) $1105[\mathrm{M}+\mathrm{H}]^{+}, 1127[\mathrm{M}+\mathrm{Na}]^{+}, 1143$ 
$[\mathrm{M}+\mathrm{K}]^{+}, 553[\mathrm{M}+2 \mathrm{H}]^{2+}, 564[\mathrm{M}+\mathrm{H}+\mathrm{Na}]^{2+}, 575[\mathrm{M}+2 \mathrm{Na}]^{2+} . \quad m / z(\mathrm{MALDI}-\mathrm{TOF}$ (Kratos) MS) $1105[\mathrm{M}+\mathrm{H}]^{+}, 1127[\mathrm{M}+\mathrm{Na}]^{+}$, calculated $\mathrm{M}_{\mathrm{w}}=1104.51 . \mathrm{GPC} ; \mathrm{M}_{\mathrm{w}}=$ $1180, \mathrm{Pd}=1.00$.
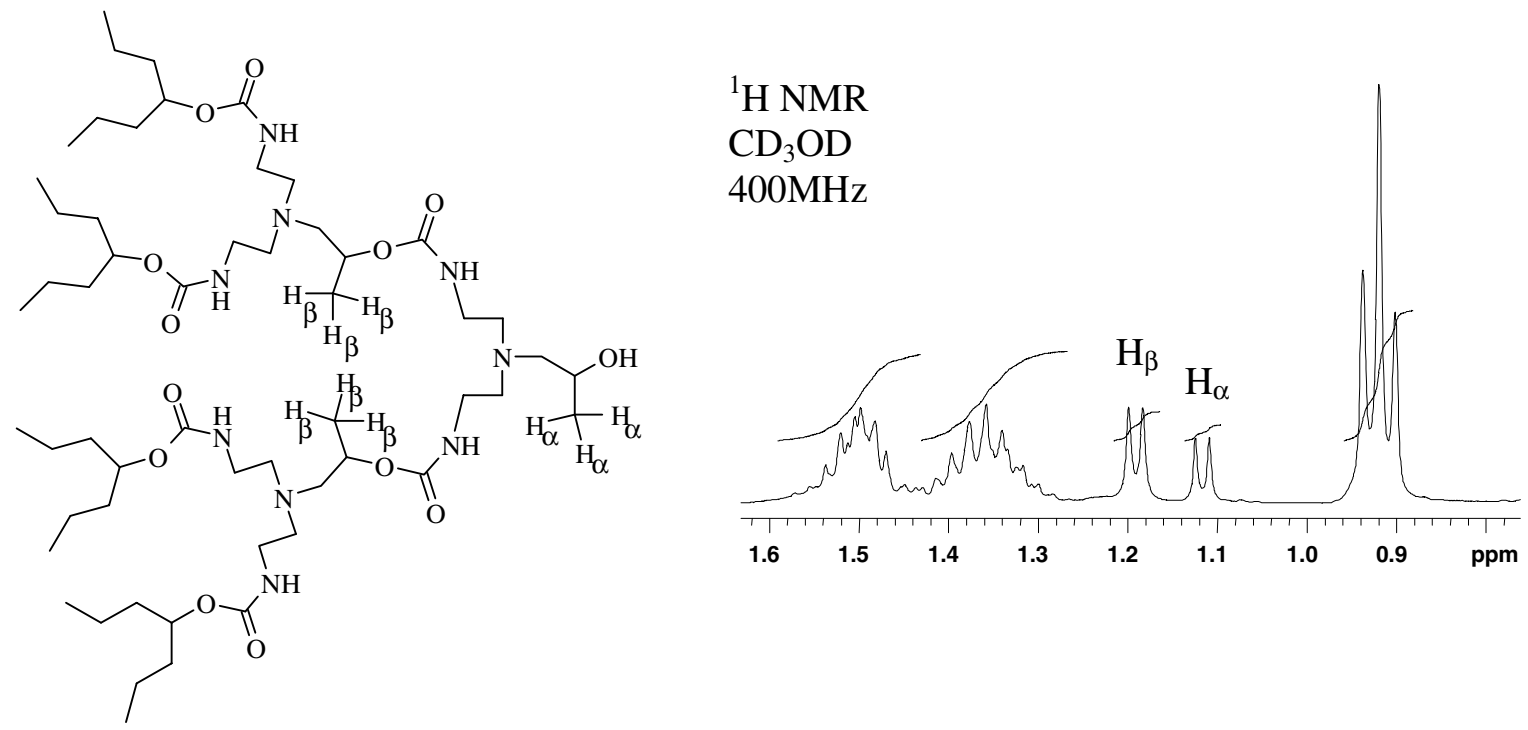

Methyl group hydrogens of G2-[4-Hept-AEAP 2$] \mathrm{OH}$
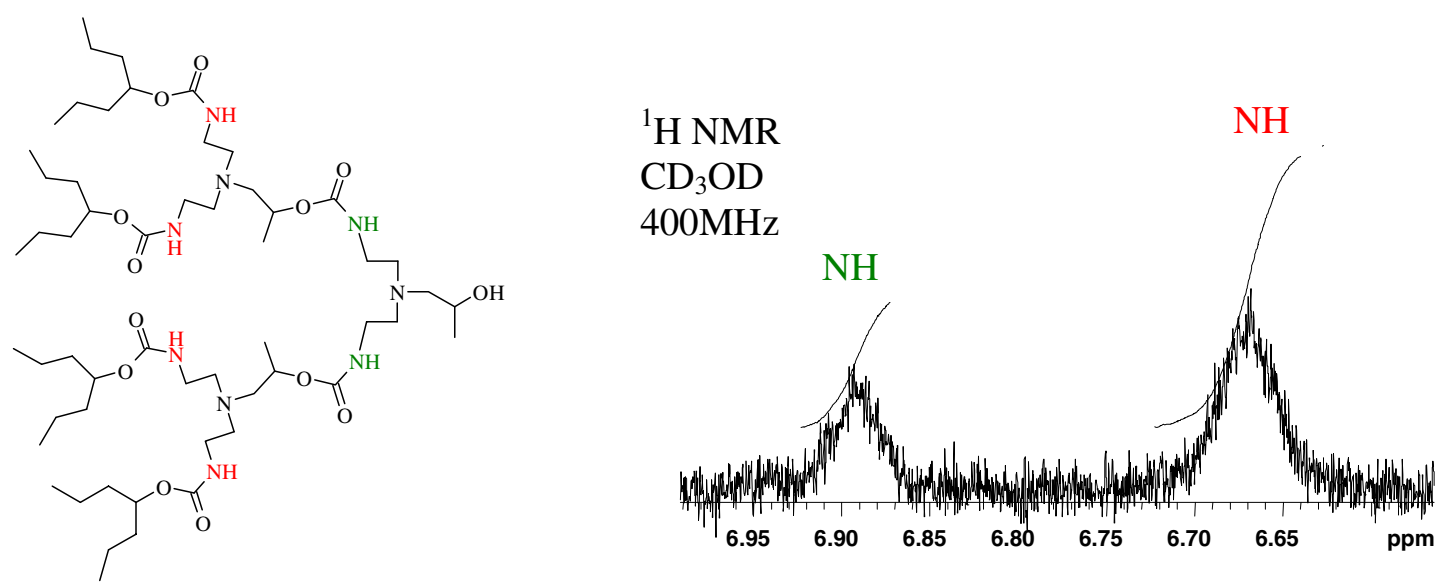

Urethane $\mathrm{N}-\mathrm{H}$ resonances of G2-[4-Hept-AEAP 2$] \mathrm{OH}$ 


\section{${ }^{1} \mathrm{H}$ NMR Spectrum of G2-[4-Hept-AEAP 2 ]OH}

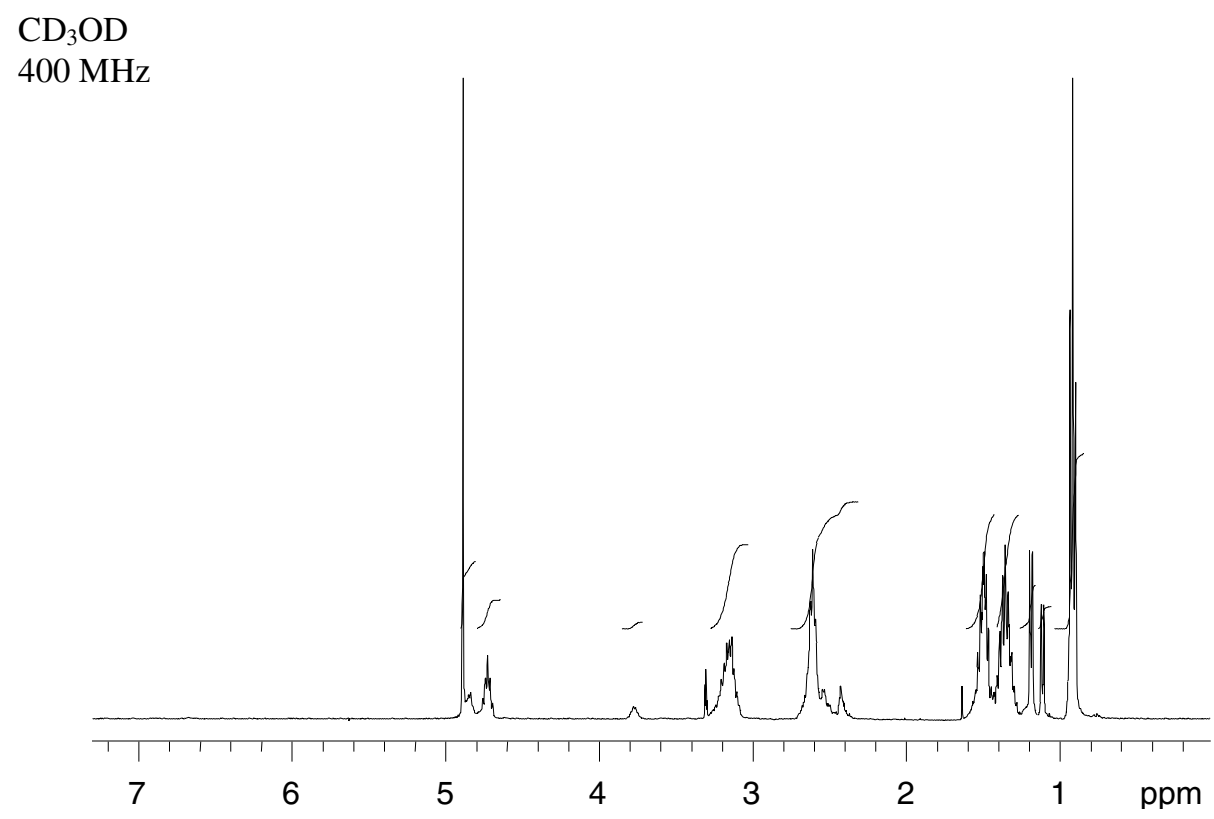




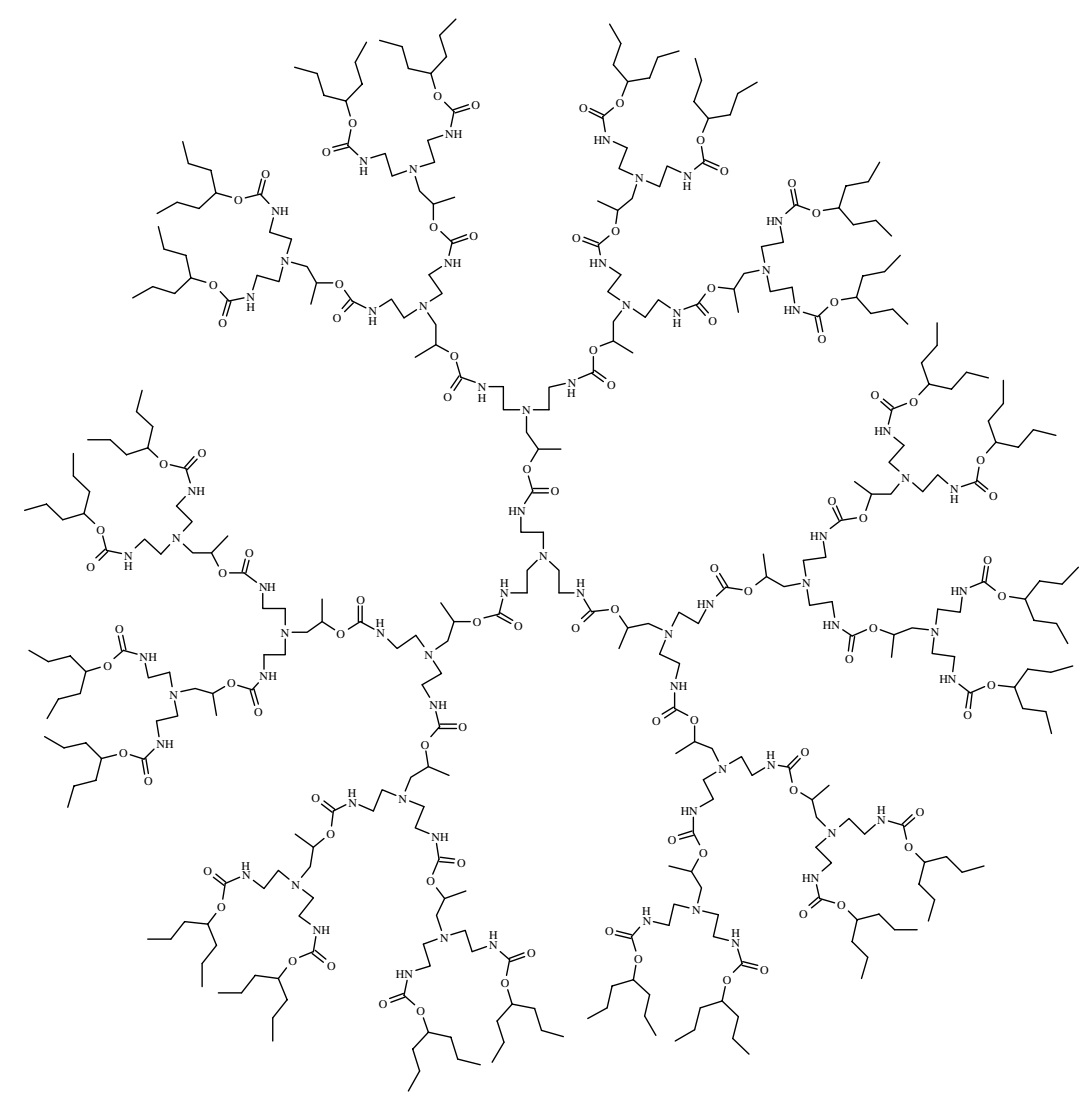

Synthesis of G3-[4-Hept-AEAP 3 TAEA:

CDI $(0.57 \mathrm{mmol})$ was added to a stirred solution of G3-[4-Hept-AEAP 3 ]OH $(0.47$ $\mathrm{mmol})$ in toluene $(40 \mathrm{~mL})$ and the mixture was heated at $60^{\circ} \mathrm{C}$ for 4 hours. Subsequently, the reaction mixture was analysed by ${ }^{1} \mathrm{H}$ NMR spectroscopy and interpretation of the spectrum indicated no evidence of the presence of starting materials (i.e. G3-[4-Hept-AEAP 3 ] OH or CDI). TAEA $(0.16 \mathrm{mmol})$ was added to the solution and the mixture was heated for 1 day at $60^{\circ} \mathrm{C}$. The reaction mixture was concentrated in vacuo and redissolved in $\mathrm{CH}_{2} \mathrm{Cl}_{2}(100 \mathrm{~mL})$. The organic phase was subsequently washed with water ( 3 x $100 \mathrm{~mL}$ ), dried over $\mathrm{MgSO}_{4}$ and the solvent removed using the rotary evaporator. The yellow oil obtained was purified by column chromatography (silica gel, eluting with EtOAc:MeOH 100:5 increasing to EtOAc:MeOH 100:10). The colourless oil obtained was purified further by preparative GPC (Biobeads, eluting with toluene) to give compound G3-[4-Hept$\mathrm{AEAP}_{3}$ ]TAEA as an extremely sticky oil $(280 \mathrm{mg}, 24 \%) . \mathrm{T}_{\mathrm{g}}=19.4^{\circ} \mathrm{C}$. Found C, 57.37; H, 9.50; N, 11.88\%. $\mathrm{C}_{366} \mathrm{H}_{711} \mathrm{O}_{90} \mathrm{~N}_{67}$ requires, $\mathrm{C}, 58.68 ; \mathrm{H}, 9.57 ; \mathrm{N}, 12.53 \%$. ${ }^{13} \mathrm{C}$ NMR $\left(100 \mathrm{MHz}, \mathrm{CD}_{3} \mathrm{OD}\right) \delta 14.6,19.1,19.7,40.0,40.1,55.7,61.0,70.7,75.2$, 158.7, 158.8, 159.3. ${ }^{1} \mathrm{H}$ NMR $\left(400 \mathrm{MHz}, \mathrm{CD}_{3} \mathrm{OD}\right) \delta 0.92(\mathrm{t}, \mathrm{J}=7.2 \mathrm{~Hz}, 144 \mathrm{H}), 1.20(\mathrm{~d}$, $\mathrm{J}=6 \mathrm{~Hz}, 63 \mathrm{H}), 1.36(\mathrm{~m}, 96 \mathrm{H}), 1.50(\mathrm{~m}, 96 \mathrm{H}), 2.51-2.66(\mathrm{~m}, 132 \mathrm{H}), 3.12-3.20(\mathrm{~m}$, $90 \mathrm{H}), 4.74(\mathrm{~m}, 24 \mathrm{H}), 4.85$ (m, obscured by water peak, 9H), 6.46 (s, br, $\left.\mathrm{OC}(\mathrm{O}) \mathrm{NHCH}_{2}\right), 6.76$ (s, br, $\left.\mathrm{OC}(\mathrm{O}) \mathrm{NHCH}_{2}\right) . \mathrm{m} / \mathrm{z}(\mathrm{ES} \mathrm{MS}) 7511.4[\mathrm{M}+\mathrm{Na}]^{+}, 7527.5$ 
$[\mathrm{M}+\mathrm{K}]^{+}$, calculated $\mathrm{M}_{\mathrm{w}}=7490.96$ and an impurity at 5063.6 corresponding to the two-armed dendrimer. GPC; $\mathrm{M}_{\mathrm{w}}=6410, \mathrm{Pd}=1.01$.

${ }^{1} \mathrm{H}$ NMR Spectrum of G3-[4-Hept-AEAP 3 ]TAEA:

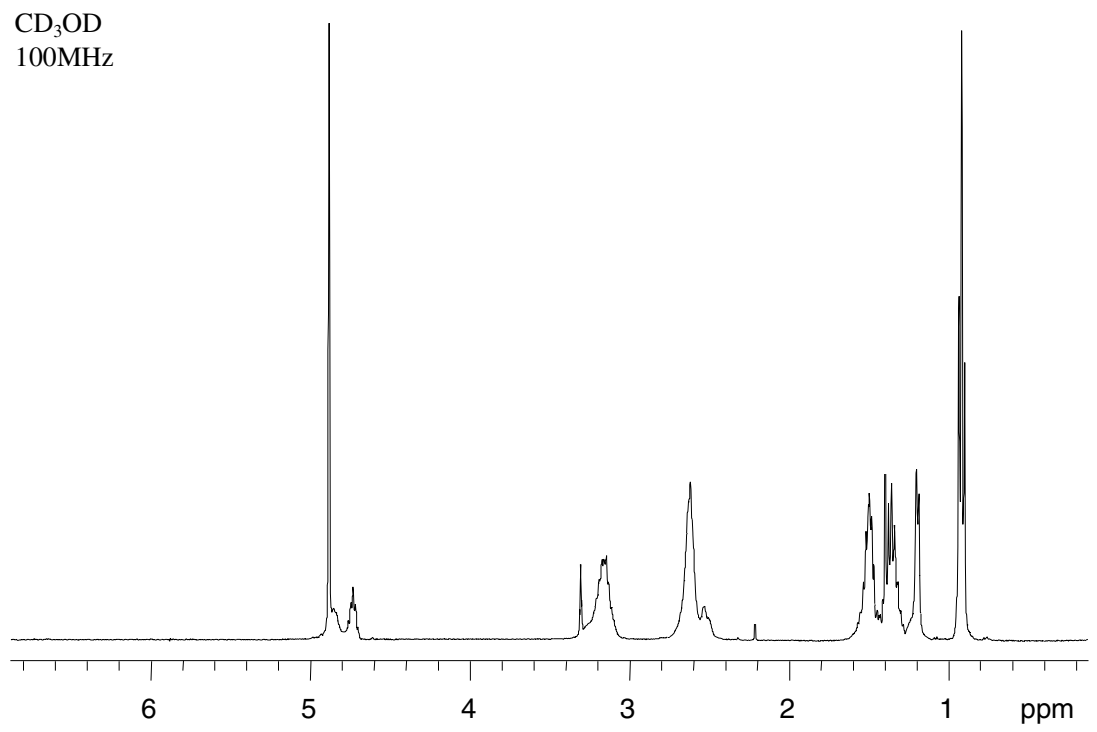

MALDI-TOF (Voyager) mass spectrum of G3-[4-Hept-AEAP 3 TAEA

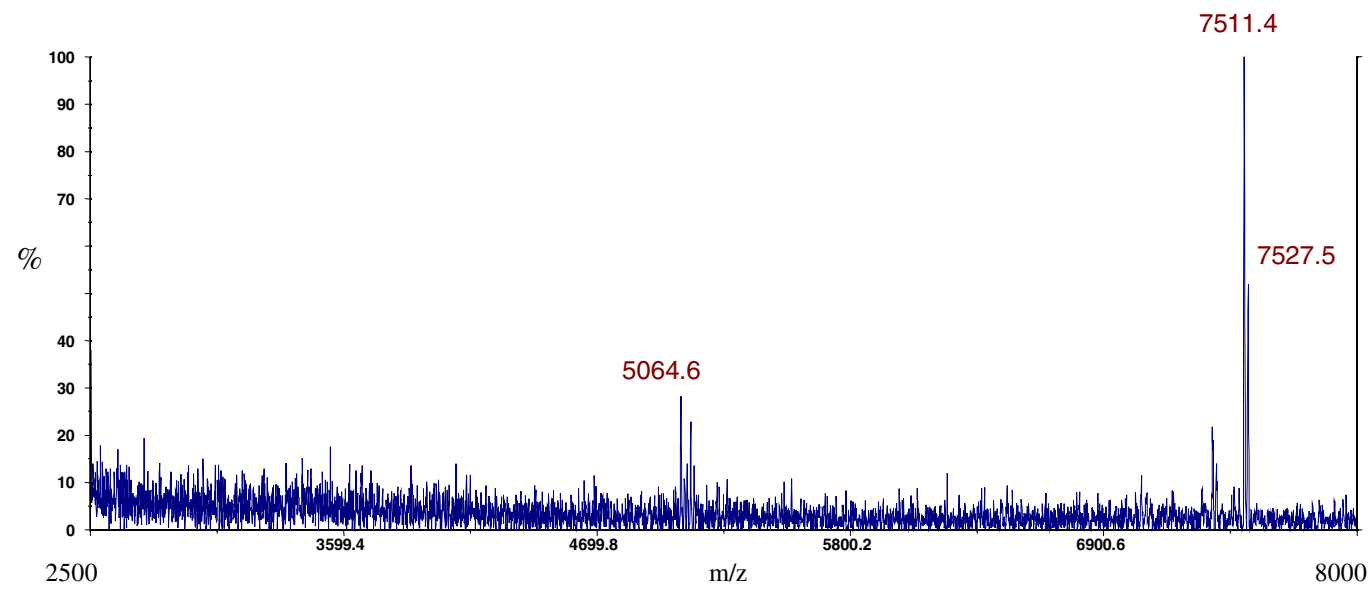

